\title{
Flow measurements in sewers based on image analysis: automatic flow velocity algorithm
}

\author{
D. Jeanbourquin, D. Sage, L. Nguyen, B. Schaeli, S. Kayal, D. A. Barry \\ and L. Rossi
}

\begin{abstract}
Discharges of combined sewer overflows (CSOs) and stormwater are recognized as an important source of environmental contamination. However, the harsh sewer environment and particular hydraulic conditions during rain events reduce the reliability of traditional flow measurement probes. An in situ system for sewer water flow monitoring based on video images was evaluated. Algorithms to determine water velocities were developed based on image-processing techniques. The imagebased water velocity algorithm identifies surface features and measures their positions with respect to real world coordinates. A web-based user interface and a three-tier system architecture enable remote configuration of the cameras and the image-processing algorithms in order to calculate automatically flow velocity on-line. Results of investigations conducted in a cSO are presented. The system was found to measure reliably water velocities, thereby providing the means to understand particular hydraulic behaviors.
\end{abstract}

D. Jeanbourquin

L. Nguyen

B. Schaeli

S. Kayal

D. A. Barry

L. Rossi (corresponding author)

Ecological Engineering Laboratory (ECOL),

Ecole Polytechnique Fédérale de Lausanne (EPFL), Lausanne,

Switzerland

E-mail: luca.rossi@epfl.ch

D. Sage

Biomedical Imaging Group (BIG)

Ecole Polytechnique Fédérale de Lausanne (EPFL), Lausanne,

Switzerland

Key words $\mid$ combined sewer overflow, flow measurement, homography, image processing, video monitoring, water level measurement

\section{INTRODUCTION}

Long-term measurement of sewer flows is a difficult task. The sewer environment is harsh, with humidity of $100 \%$ and possibly corrosive gases. Flow measurement devices in contact with wastewater are at risk of being destroyed, especially by the rapid increases in water elevation and flow rate due to storms. Access entails risk for individuals charged with replacing or maintaining equipment.

The most common means to measure flow is the Doppler meter based on two data, the height of the water (measured by pressure difference) and the flow velocity measured by the Doppler shift principle (Jensen 2004). Such meters must be placed inside dirty water and hence are not reliable for long-term measurements. An alternative solution avoiding water contact involves measurement of the water height (e.g. using an ultrasonic probe), from which the flow velocity is determined using a rating curve. However, the rating curve, being a global hydraulic modelbased curve, is incapable of capturing local hydraulic effects and hence in general cannot provide accurate results.

Here, we propose an alternative to measure the surface water velocity using video analysis. The video clips are acquired by a camera mounted in a waterproof case; illumination is provided by an infrared illumination device positioned above the sewer channel. None of the hardware comes in contact with the water. Image-processing algorithms are presented for calculating the water flow rate. This is the first video-based implementation of an automatic flow measurement system. LSPIV techniques (Large Scale Particle Image Velocimetry) have been applied to open channels and rivers (Jodeau et al. 2008), but the conditions are rather different. The addition of tracer particles is not necessary in sewers due to floating waste. Moreover, measurements in rivers with cameras were performed for a very limited duration only and were not designed for extended periods. In the open, the illumination change between day and night is difficult to control. In a closed environment, such as a sewer, the light conditions are constant.

These developments are part of a complete sewer monitoring system called HydroPix Monitoring (Nguyen et al. 2009). The HydroPix Monitoring system is a robust visionbased package that integrates several modules and features: visual analysis of hydraulic behavior, on-line water level 
measurements, wastewater velocity measurements, automatic alarm system for particular events (overflows, flood risks, etc.), database management (images, events, measurements, etc.), remote configuration and data visualization. The system was developed considering the accessibility and difficult conditions within sewers, and is able to take measurements autonomously for long periods with minimum maintenance. Previously, Nguyen et al. (2009) focused on water level measurement. Here, velocity measurements are considered in detail.

\section{$\overline{\text { METHODOLOGY }}$}

The flow rate $D$ is given by:

$D=P(h) \cdot V\left[m^{3} / S\right]$

where $V(\mathrm{~m} / \mathrm{s})$ is the water velocity and $P(h)\left(\mathrm{m}^{2}\right)$ describes the water channel geometry as it varies with the water level $h(\mathrm{~m}) . P(h)$ can be deduced from the construction plans so computation of $D$ is based on $h$ and $V$, which are obtained from image analysis.

Images are captured with infrared cameras. In the following sections the analyses based on the captured images are outlined.

\section{Water level measurement}

The water-air interface is detected using an infrared camera image. The advantages of this vision-based system have been demonstrated (Khorchani \& Blanpain 2004; Nguyen et al. 2009).

\section{Flow velocity measurement}

There are two approaches for measuring flow velocity: lowlevel motion estimation or feature-point tracking. The former operates at the pixel level in the spatio-temporal domain, with several image analysis methods including optical flow (Horn \& Schunck I98I), block-matching, correlation and the spatio-temporal orientation method (Jähne 1993). Such approaches are not suitable for sewer images since the wastewater primarily exhibits homogeneous intensity in grayscale images (infrared images are grayscale). Moreover, low-level methods require a high similarity from one frame to the next frame to establish pixel correspondences. In sewers, cameras cannot run at sufficiently high frame rates due to low light conditions, making low-level methods inapplicable in practice.

Feature-point tracking is used when the image sequence consists mainly of moving objects over a static or quasihomogenous background. In sewers, a few moving objects are often present on the water surface. These small objects appear as brighter or darker spots in the images. We designed an image-analysis system to track them over an image sequence. The algorithm first detects bright areas (called features) on individual images, which are assumed to have the same velocity as the flow, although correction factors can be used (Larrarte 2006).

In sewers the camera position is imposed by local access considerations, generating issues of perspective and geometric distortions in the images. Thus, for accurate results the image analysis must suitably correct the images. Moreover, the velocity is determined on the surface water, whose height changes temporally. The variable-height homography transformation dynamically accounts for water level changes, as described below.

\section{Data acquisition}

The HydroPix system acquires images using three cameras: one for the measurement of water level, one for the computation of water velocity and one to have an overview of the combined sewer overflow (CSO). AXIS 221 Network cameras were selected as they adapt the color depth of the images and the acquisition frame rate to the lighting conditions. They typically produce color sequences at $40 \mathrm{fps}$ for optimal illumination, but switch to variable frame rate black and white images (in the infrared spectrum) with poor lightening. They are PoE (Power-over-Ethernet) IP cameras, which enable both power and data to go through the same cable. The use of standard Ethernet connectors makes the system independent of a specific camera manufacturer. The light sources used are low-power, water-resistant infra-red LEDs. An important benefit for sewers is that, in contrast to visible light, infrared has the benefit of not attracting insects. With suitably placed LEDs, a $25 \mathrm{fps}$ frame rate was achieved. The cameras are installed in IP67 waterproof cases. Cameras and LEDs are connected to a connectivity box containing a power supply and server PC for image processing.

Algorithms were implemented as a National Instrument LabVIEW Service. Images are saved on the PC and automatically processed by the different algorithms. Results are saved in a PostgreSQL database and are automatically periodically uploaded to remote sites using an internet connection. HydroPix also manages an alarm system, which 
automatically uploads and saves images under specified conditions. Usually, network connectivity is unavailable in sewers, so monitors (defined as in situ monitoring system in our context) rely on the UMTS network, using an external antenna to transmit data from the PC server. Finally, the PC server provides a web-based user interface for managing and configuring the monitors. The total cost of the HydroPix Monitoring prototype including software licenses and hardware equipment is approximately US\$12K.

\section{Water velocity method}

The water velocity measurement techniques developed in our application are based on PIV (Particle Image Velocimetry) (Chetverikov 200I) approaches or feature-based tracking. The algorithm to obtain the surface flow velocity is summarized in Figure 1, and consists of two steps. First, the feature detection and tracking step allows for particle velocity estimation in pixels per frame. Second, the variable-height homography technique transforms this velocity into realworld (m/s) units.

\section{Feature detection and tracking}

Due to noise (light reflections, particles, data compression, etc.), Gaussian smoothing is applied on all images at the

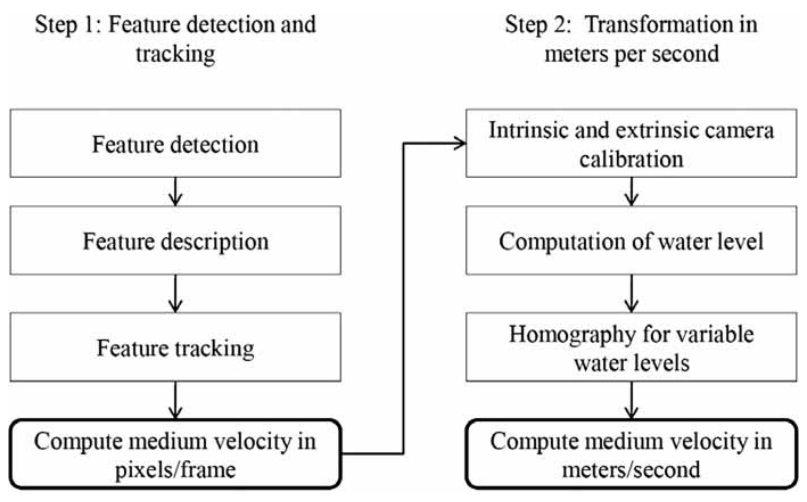

Figure 1 | Overview of the algorithms to estimate surface flow velocity. beginning of the process. This smoothing is tuned in order to attenuate the noise without removing small particles of interest. A background image is then estimated by a pixelwise temporal median filter applied to a small sequence (typically 100 images). This estimated background image is subtracted from each image to accentuate moving particles. Then, an adaptive threshold is applied to get binary images containing moving objects. Finally, the binary images are eroded to suppress the particles composed of only a few pixels (Figure 2).

Particles $P_{k}$ within the images are detected, labeled and described (Feature description). Here, the shape features pertinent to identify a particle are the area $A\left(P_{k}\right)$ and circularity $C\left(P_{k}\right)$.

A matching coefficient between each particle in an image $F_{t}$ with each particle in the next image $F_{t+1}$ is computed (Feature tracking). This matching coefficient (cost function, $\zeta$ ) is a weighted sum of three normalized terms:

$$
\begin{aligned}
\zeta\left(P_{i, t}, P_{j, t+1}\right)= & \lambda_{\mathrm{a}}\left\|\frac{A\left(P_{i, t}\right)-A\left(P_{j, t+1}\right)}{\max \left(A\left(P_{i, t}\right), A\left(P_{j, t+1}\right)\right)}\right\| \\
& +\lambda_{c}\left\|C\left(P_{i, t}\right)-C\left(P_{j, t+1}\right)\right\| \\
& +\lambda_{\mathrm{d}}\left(1-\left\langle\overrightarrow{P_{1, t} P_{j, t+1}} \cdot \overrightarrow{V_{f}}\right\rangle\right)
\end{aligned}
$$

The first two terms account for $A\left(P_{k}\right)$ and $C\left(P_{k}\right)$, while the third term is the variation of the displacement vector (normalized) relative to the normalized flow direction vector $V_{f}$ corresponding to the channel orientation (Jeanbourquin 2009). The $\lambda$ 's are tuning parameters, with $\lambda_{\mathrm{a}}$ and $\lambda_{c}$ providing weights relevant to particle shapes and $\lambda_{d}$ the weight for constraining particle motion.

The goal of feature matching is to find the minimal cost functions between particles in two consecutive images. Figure 3 shows particles in two sequential frames that are considered as a bipartite graph. The process to find acceptable matching between particles is performed in two steps. First, for every particle $P_{i, t}$ in frame $F_{t}$, the cost function is calculated for all particles $P_{j, t+1}$ in $F_{t+1}$ to deduce the best match. (a)

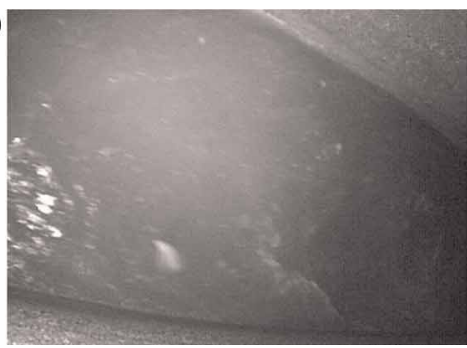

(b)

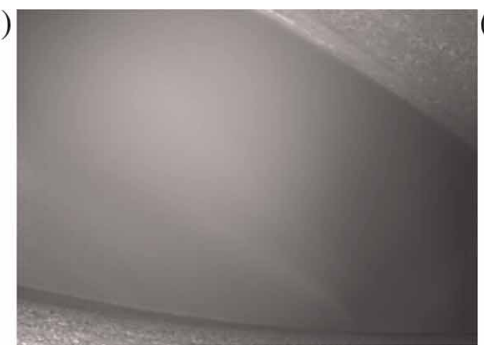

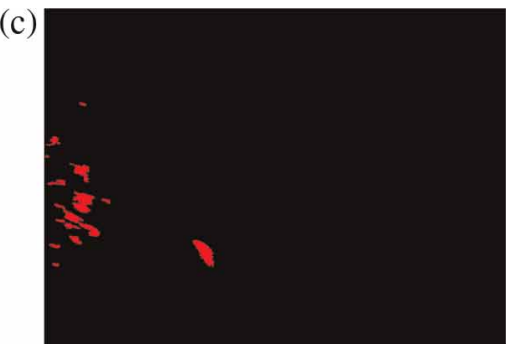

Figure 2 | Image analysis for particle detection. (a) Original infrared image; (b) background estimation; (c) binary image with possible particles for velocity measurement. 

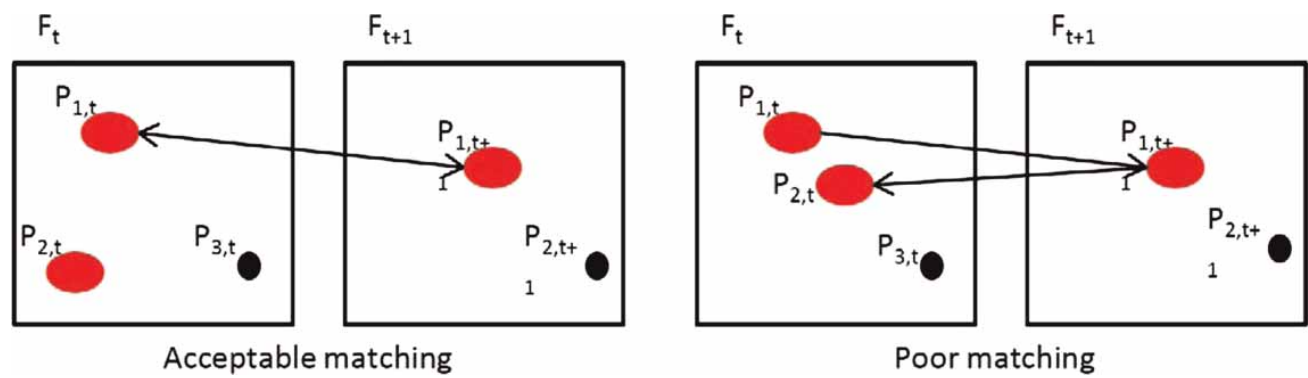

Figure 3 Bipartite graph concept. Left: $P_{1, t}$ matched with $P_{1, t+1}$ and vice versa, so acceptable. Right: $P_{1, t}$ matched with $P_{1, t+1}$ but $P_{1, t+1}$ matched with $P_{2, t}$, so unacceptable.

Next, the same computation is reversed, giving a second best match for $F_{t+1}$ to $F_{t}$. If the two best matches are the same they are used in the next step to determine the water displacement. Thus, the algorithm then tracks particles in subsequent images (typically 5 frames at $25 \mathrm{fps}$ ). Outliers are avoided by tracking particles over more than two images.

The center of mass of each tracked particle in each image and the frame rate are used to determine the particle velocity. This process is usually conducted on video sequences of eight seconds at a rate of $25 \mathrm{fps}$. The median value of individual particles is used to estimate the surface flow velocity.

\section{Variable-height homography}

Camera calibration is needed to find the real-world velocity from the image-based pixels/second velocity. This procedure involves determining the parameters governing the projection of real-world points to image pixels. These parameters are separated into two categories: intrinsic parameters that depend on the camera (objective type, focal length, CCD captor size) and extrinsic parameters that are related to the camera position. The intrinsic parameters can be described by the pinhole camera model (Zhang 2000), which considers the relation between the 3D coordinates of a point and its projection onto the image plane of an ideal camera. However, camera lenses cause a nonlinear distortion effect in images, which is removed using a distortion model (Bouguet 2008). The OpenCV library (Open Source Computer Vision, OpenCV 2009) was used to account for intrinsic camera calibration and image distortion.

The extrinsic parameters relate a chosen system of realworld coordinates to the camera coordinates. This transformation, called homography, is composed of a rotation $R$ and a translation $T$ representing a linear transformation between two projective planes (Estrada et al. 2004). It is used to project a plane in the real world to the image plane. Pixel coordinates of a point in an image can be located in the real world coordinate system. This transformation has been successfully used to estimate the water levels based on image analysis (Nguyen et al. 2009). Here, however, the water surface is not static, causing the homography to change with the flow height. A variable-height homography was therefore developed and implemented (Jeanbourquin 2009). Starting from a 'classical' homography (Equation (3)):

$$
\begin{aligned}
& {\left[\begin{array}{c}
S_{u} \\
S_{v} \\
S
\end{array}\right] }=\left[\begin{array}{ccc}
\alpha_{u} & 0 & u_{0} \\
0 & \alpha_{v} & v_{0} \\
0 & 0 & 1
\end{array}\right]\left[\begin{array}{lll}
r_{11} & r_{12} & t_{x} \\
r_{21} & r_{22} & t_{y} \\
r_{31} & r_{32} & t_{z}
\end{array}\right]\left[\begin{array}{c}
x_{w} \\
Y_{w} \\
1
\end{array}\right] \\
&=H\left[\begin{array}{c}
X_{w} \\
Y_{w} \\
1
\end{array}\right]
\end{aligned}
$$

where $S_{u}, S_{v}$ and $S$ are the image coordinates (pixels), $u_{0}$ and $v_{0}$ are the coordinates (pixels) of the projection of the optical center of the image, $r_{i j}$ and $t_{x, y, z}$ represent the rotation $R$ and translation $T$ parameters respectively, $X_{w}, Y_{w}$ are the coordinates attached to the camera and $H$ represents the $3 \times 3$ homography matrix. The determination of the parameters of $H$ is made numerically; with at least four correspondence points in the image plane and in the real-world coordinate system. This matrix is computed by an optimization algorithm (Kovesi 2009).

If $Z_{w}$ varies over time $\left(Z_{w} \neq 0\right)$, Equation (3) becomes:

$$
\left[\begin{array}{c}
S_{u} \\
S_{v} \\
S
\end{array}\right]=\left[\begin{array}{ccc}
\alpha_{u} & 0 & u_{0} \\
0 & \alpha_{v} & v_{0} \\
0 & 0 & 1
\end{array}\right]\left[\begin{array}{llll}
r_{11} & r_{12} & r_{13} & t_{x} \\
r_{21} & r_{22} & r_{23} & t_{y} \\
r_{31} & r_{32} & r_{33} & t_{z}
\end{array}\right]\left[\begin{array}{c}
x_{w} \\
Y_{w} \\
Z_{w}
\end{array}\right]
$$

And by developing the right term of Equation (4), the result is given by Equation (5):

$$
\left[\begin{array}{c}
S_{u} \\
S_{v} \\
S
\end{array}\right]=\left[\begin{array}{ccc}
\alpha_{u} & 0 & u_{0} \\
0 & \alpha_{v} & v_{0} \\
0 & 0 & 1
\end{array}\right]\left[\begin{array}{l}
r_{11} * X_{w}+r_{12} * Y_{w}+r_{13} * Z_{w}+t_{x} \\
r_{21} * X_{w}+r_{22} * Y_{w}+r_{23} * Z_{w}+t_{y} \\
r_{31} * X_{w}+r_{32} * Y_{w}+r_{33} * Z_{w}+t_{z}
\end{array}\right]
$$


Hence, the new homography $H^{\prime}$, in function of the height (component $Z_{w}=h$ ) is (Jeanbourquin 2009):

$H^{\prime}=\left[\begin{array}{ccc}\alpha_{u} & 0 & u_{0} \\ 0 & \alpha_{v} & v_{0} \\ 0 & 0 & 1\end{array}\right]\left[\begin{array}{ccc}r_{11} & r_{12} & r_{13} * h+t_{x} \\ r_{21} & r_{22} & r_{23} * h+t_{y} \\ r_{31} & r_{32} & r_{33} * h+t_{z}\end{array}\right]$

Finally, the real-world coordinates of points in the image given in pixels are given by Equation (7):

$\left[\begin{array}{c}X_{w} \\ Y_{w} \\ 1\end{array}\right]=H^{\prime-1}\left[\begin{array}{c}S_{u} \\ S_{v} \\ S\end{array}\right]$

The intrinsic and extrinsic parameters were calibrated by measuring the size and deformation of a chessboard texture plane at different positions and angles. This chessboard is first used in the laboratory for intrinsic camera calibration. It is then used once the cameras are fixed in the sewer to determine the extrinsic parameters. The calibration process is conducted using a dedicated function in the web-based user interface.

Finally, note that the precision of the velocity estimate depends on that of the water level height. This height can be measured using an ultrasonic probe or, preferably, within HydroPix using the existing water level algorithm of Nguyen et al. (2009).

\section{RESULTS AND DISCUSSION}

\section{Velocity measurement validation in pixels/frame}

Preliminary tests were performed with image sequences, without camera calibration, with velocities computed in pixels/frame. Two different cameras were used, a PROSILICA GC 640C and an AXIS 221. The measurement reference was obtained manually by identifying particles over an image sequence, with the velocity determined by counting the displacement in pixels between images. Figure 4 presents a sequence, captured by the PROSILICA camera, in which particles were detected and tracked.

The image sequences taken by the PROSILICA camera (200 images, $40 \mathrm{fps}, 648 \times 488$ pixel resolution) led to a reference velocity estimate of 3.28 pixels/frame. Five different videos were taken sequentially in the Lausanne wastewater treatment plant. No obvious signs of velocity variations were observed during the filming, so the water flow rate is assumed to be constant. Estimate velocities and relative errors are shown in Table 1.

The results in Table 1 validate the algorithm operation. The maximum error is less than $10 \%$; its mean value is about 5.8\%. These inaccuracies come from several factors. First, the water surface velocity near a channel edge is reduced due to wall effects. Consequently, the velocity measured depends on the position of the tracked particles. Another possible error comes from the particle trajectories, which are not aligned with the flow direction. Finally, the reference computed for the velocity was determined by hand and cannot be considered as the true value.

Another set of tests was performed with an AXIS 221 camera using data from a Lausanne sewer (21 images, $40 \mathrm{fps}, 640 \times 367$ pixel resolution). Results were similar to

Table 1 | Results of velocity measurements in pixels/frame with the PROSILICA camera

\begin{tabular}{lll} 
Video number & Velocity (pixels/frame) & Relative error (\%) \\
\hline 1 & 3.6 & 9.8 \\
2 & 3.53 & 7.6 \\
3 & 3.22 & 1.8 \\
4 & 3.41 & 3.9 \\
5 & 3.09 & 5.8 \\
\hline
\end{tabular}

(a)

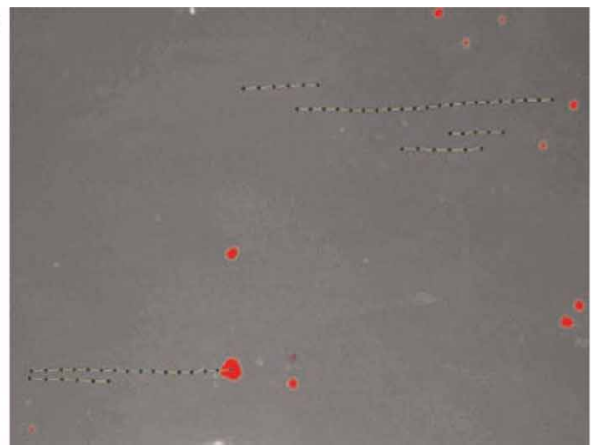

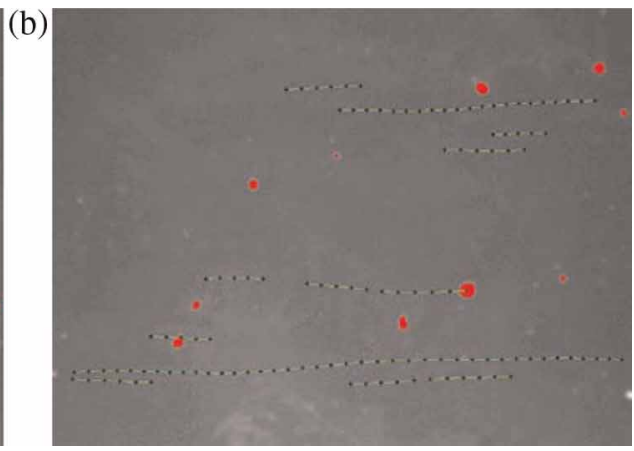

Figure 4 Particle tracking on an image sequence, in which candidate particles are highlighted. The lines correspond to particle tracking over the image sequence. Dots represent matching correspondences between sequential images. 


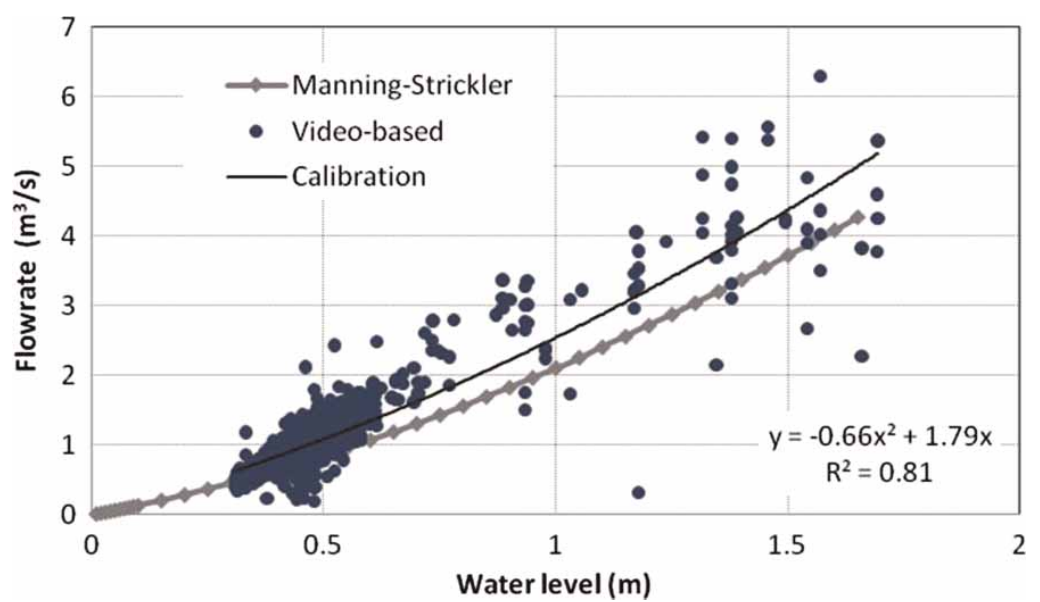

Figure 5 Rating curve established for the Berna CSO. The gray line is the theoretical rating curve (Manning-Strickler formula, with local characteristics) validated with hydraulic simulation (BG 2007). The dots refer to HydroPix-estimated surface velocities. The black line (Flow rate $=1.79 x-0.66 x^{2}$, where $x$ is water level; $R^{2}=0.81$ ) is a second-order polynomial fit of measurement points.

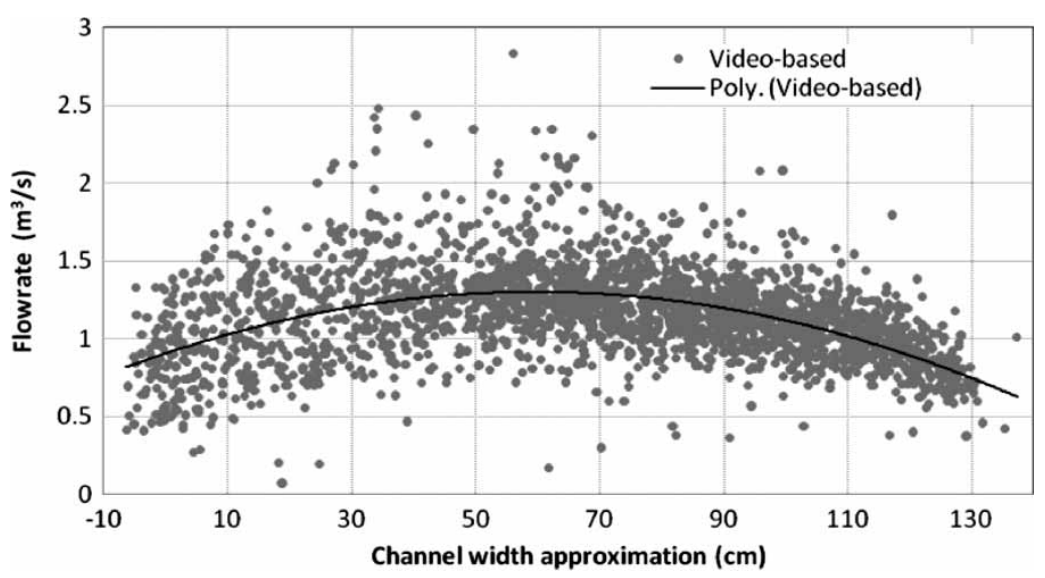

Figure 6 | Cross section of water velocities for water levels between 40 and $60 \mathrm{~cm}$ (dry-weather). The global shape of the curve (trend line in black) is typical of theoretical velocity profiles in channels (Graf \& Altinakar 1995).

those in Table 1, although the average relative error was reduced to $2.7 \%$.

\section{In situ validation}

A HydroPix Monitor system was installed in Berna CSO (Lausanne). This CSO is 20-m long and is expected to discharge at a water level of $1.63 \mathrm{~m}$, corresponding to a flow rate of $4.19 \mathrm{~m}^{3} / \mathrm{s}$. An AXIS 221 camera was installed above the flow. Videos of about eight seconds at $25 \mathrm{fps}$ were filmed every $30 \mathrm{~min}$ for 6 weeks, followed by off-line image processing. The computation time for an image sequence is about eight seconds with an Intel Core 2 $2.13 \mathrm{GHz}$ Duo Processor. The water level was measured both with an ultrasonic flow meter (Teledyne ISCO 4210) and with the HydroPix method (Nguyen et al. 2009), with good agreement between the two. The rating curve was calculated (Figure 5) and compared with a theoretical rating curve based on a Strickler law and hydraulic modeling (BG 2007). Despite outliers, results are promising and represent well the hydraulic behavior. As the position of each particle was also recorded, it is possible to illustrate the velocity of particles at different channel widths (Figure 6). The parabolic shape shown in Figure 6 agrees well with the theoretical velocity profile for a channel (Graf \& Altinakar 1995).

\section{CONCLUSIONS}

The feature tracking approach has been demonstrated to be a practical technique for sewer flow velocity measurements. 
Separate steps in the HydroPix approach were validated separately. For example, results obtained for velocities in pixels/frame showed the feasibility of the algorithms used. Results can be considered as reliable, with an error upper bound of about 10\%. Image data from long term CSO monitoring permitted estimation of the channel rating curve based. Results were very encouraging, being in agreement with theoretical and modeled hydraulic behavior. The HydroPix system allows also estimation of the surface flow velocity at different positions in a sewer cross section. One limitation of the system is that velocities are measured at the water surface, and is not equal to the average flow rate. Depending on flow conditions, the average velocity can be determined with increased accuracy by using the Manning-Strickler equation or other hydraulic formulas. Therefore, correction factors based on local hydraulic conditions may be applied.

Tests are planned for other CSO configurations to evaluate the approach under different flow conditions. Based on current results, we conclude that image-based measuring devices represent a feasible means to measure and better understand sewer flows and hydraulics.

\section{ACKNOWLEDGEMENTS}

The authors thank the Swiss Commission for Technology and Innovation (KTI/CTI CTI 8934.1 PFIW-IW) for financial support, the Etrinex SA Company (industrial partner of the project) and the City of Lausanne for their help in conducting measurements in the sewer system of the city.

\section{REFERENCES}

BG 2007 Ville de Lausanne, Service d'Assainissement: Calculs Hydrauliques des Déversoirs d'Orage. Report of BG, Consulting Engineers, ref. 6505.01-RN011b/Esd/Mob/Swr, Lausanne, Switzerland.

Bouguet, J. Y. 2008 Camera Calibration Toolbox for Matlab California Institute of Technology (Caltech), Pasadena (CA). Available from: www.vision.caltech.edu/bouguetj/calib_doc/ (accessed 14 July 2011).

Chetverikov, D. 200I Particle image velocimetry by feature tracking. In Computer Analysis of Images and
Patterns, Vol. 2124/2001. Springer Verlag, Berlin/ Heidelberg.

Estrada, F. J., Jepson, A. D. \& Fleet, D. 2004

Planar Homographies. Lecture notes,

University of Toronto, Canada. Available from: www.cs. utoronto.ca/ strider/vis-notes/tutHomography04.pdf (accessed 14 July 2011).

Graf, W. H. \& Altinakar, M. S. I995 Hydrodynamique: Une introduction. In: Traité de génie civil, Vol. 14, PPUR eds. Presse Polytechnique et Universitaires Romandes, Lausanne, Switzerland.

Horn, B. K. P. \& Schunck, B. G. I98I Determining Optical Flow. Artificial Intelligence 17, 185-203.

Jähne, B. I993 Spatio-Temporal Image Processing. In Lectures Notes in Computer Science, Vol. 751, Springer, Berlin, Germany.

Jeanbourquin, D. 2009 Implementation of an Image-Based Surface Water Velocity Measurement Algorithm. Master's Thesis EPFL, ENAC, Laboratory of ecological engineering (ECOL), Lausanne, Switzerland.

Jensen, K. D. 2004 Flow Measurements. Journal of the Brazilian Society of Mechanical Sciences and Engineering 26 (4), 400-419.

Jodeau, M., Hauet, A., Paquier, A., Le Coz, J. \& Dramais, G. 2008 Application and evaluation of LS-PIV technique for the monitoring of river surface velocities in high flow conditions. Flow Measurement and Instrumentation 19 (2), 117-127.

Khorchani, M. \& Blanpain, O. 2004 Free surface measurement of flow over side weirs using the video monitoring concept. Flow Measurement and Instrumentation 15 (2), 111-117.

Kovesi, P. D. 2009 MATLAB and octave functions for computer vision and image processing. School of Computer Science and Software Engineering. The University of Western Australia. Available from: www.csse.uwa.edu.au/ $\sim$ pk/research/matlabfns/ (accessed 14 July 2011).

Larrarte, F. 2006 Velocity fields within sewers: an experimental study. Flow Measurement and Instrumentation 17, 282-290.

Nguyen, L., Schaeli, B., Sage, D., Kayal, S., Jeanbourquin, D., Barry, D. A. \& Rossi, L. 2009 Vision-based system for the control and measurement of wastewater flow rate in sewer systems. Water Science and Technology 60 (9), 2281-2289.

OpenCV 2009 Library of programming functions for real time computer vision. Available from: http://opencv. willowgarage.com/wiki/ (accessed 14 July 2011)

Zhang, Z. 2000 A Flexible New Technique for Camera Calibration. IEEE Transactions on Pattern Analysis and Machine Intelligence 22 (11), 1330-1334. 